\title{
Understanding the Etiology and Treatment Approaches of Schizophrenia: Theoretical Perspectives and Their Critique
}

\author{
Minjeong Kim \\ School of Nursing, San Diego State University, San Diego, CA, USA \\ Email: minjeong.kim2@gmail.com
}

How to cite this paper: Kim, M. (2016) Understanding the Etiology and Treatment Approaches of Schizophrenia: Theoretical Perspectives and Their Critique. Open Journal of Psychiatry, 6, 253-261.

http://dx.doi.org/10.4236/ojpsych.2016.64030

Received: September 1, 2016

Accepted: October 21, 2016

Published: October 24, 2016

Copyright $\odot 2016$ by author and Scientific Research Publishing Inc. This work is licensed under the Creative Commons Attribution International License (CC BY 4.0).

http://creativecommons.org/licenses/by/4.0/

\section{(c) (i) Open Access}

\begin{abstract}
This paper reviewed genetic, neurodevelopmental, and neurobiological perspectives to understand the etiology and relevant treatment approaches of schizophrenia. Although genetic and neurodevelopmental theories provide a substantial contribution to the etiology of schizophrenia, neurobiological one has its dominant stance in which it can explain causative mechanisms of schizophrenia, identify targets for treatment, and predict outcomes well. According to the neurobiology, onset and course of schizophrenia are well supported by the alterations in neurotransmitters such as dopamine or serotonin. Based on this mechanism, antipsychotics have been widely used as one of the treatment approaches for schizophrenia. By following up the degree of patients' responses to antipsychotics, treatment outcomes could be evaluated or predicted. Although neurobiology seldom provides information about identification or prevention of risk factors about schizophrenia compared to genetic and neurodevelopmental approaches, it has well-established scientific foundations, ability to guide treatment, applicability across age and culture, and usefulness as a research framework.
\end{abstract}

\section{Keywords}

Schizophrenia, Theory, Neurobiology

\section{Introduction}

Schizophrenia is a severe chronic mental disorder. It is diagnosed with delusions, hallucinations, disorganized speech and behavior, and negative symptoms [1]. Schizophrenia develops during adolescence or early adulthood period, except for early-onset schizophrenia developed during school-age period which is extremely rare [2]. 12-month 
prevalence of schizophrenia is about $1.1 \%$ of the U.S. adult population [3]. Although scientific foundations about risk factors related to schizophrenia, treatment methods, and measurable outcomes, have been better established in recent years, it is still unclear how specific factors can influence the development and course of schizophrenia.

Up until now, genetic, neurodevelopmental, and neurobiological theories have been regarded as major theories explaining the etiology of schizophrenia. It is important to note that these theories should not be envisioned as being separate from each other because they emphasize relatively different focuses on the development of schizophrenia. Therefore, this paper 1) briefly described these three major theories regarding etiology of schizophrenia; 2) described how the predominant theory, neurobiological theory, helped to explain or predict the onset and course of schizophrenia; 3) described relevant treatment methods and approaches to care based on the neurobiological theory; and 4) critiqued the neurobiological theory, as to several categories.

\section{Major Theories about the Etiology of Schizophrenia}

\subsection{Genetic Theory}

Through epidemiological studies (including those with twins, in adoption settings, and with siblings), the heritability of schizophrenia has been reported as being approximately $80 \%$ to $85 \%$ [4]. Through molecular genetic studies, including candidate gene studies, genome-wide linkage studies, and genome-wide association studies (GWAS), genetic theories have contributed explanations about the development of schizophrenia [5]. In these studies, genes such as catechol-O-methyl transferase (COMT), disrupted in schizophrenia 1 (DISC1), and neuregulin 1 (NRG1) have been suggested as being the major genes involved in schizophrenia [4]. A meta-analysis study identified locus 1q2122, 12q22, and chromosomes 6, 9 and 20 as being involved symptom dimensions of schizophrenia [6]. However, there are still unanswered questions as to how genes have an impact on the development of schizophrenia [7].

\subsection{Neurodevelopmental Theory}

Along with the growing evidence of genetic contributions in schizophrenia, it has also been suggested that neurodevelopmental theory plays a pivotal role in understanding schizophrenia. This theory concerns about linkage of genetics, environmental exposures, and pathogenic occurrences [8]. Adverse environmental exposures during perinatal period such as maternal viral infections, fetal malnutrition, or extreme prematurity, may cause pathological alterations in cerebral cortex, cerebellar vermis, limbic system, brain stem, and cerebral symmetry [3] [9]. Furthermore, structural abnormalities such as lateral ventricular enlargement and third ventricle enlargement in the brain of schizophrenic patients are founded as strong evidences for neurodevelopmental origin [3].

\subsection{Neurobiological Theory}

With the development of neuroimaging, another possible explanation of the etiology of 
schizophrenia has been addressed. As per neurobiological theory, schizophrenia is regarded as the result of structural and functional abnormalities of the brain. Several domains such as the structure of the brain, physiology, chemistry, and neuropathology, are involved in this theory to explain schizophrenia. Etiological finding are as follows: 1) reductions in white matter structures, 2) functional alterations and impaired functional connectivity in prefrontal cortex, 3) reductions in neuronal and membrane integrity, and 4) neurotransmitter abnormalities [10]. Decrease in hippocampus volume is an important neuropathology finding of schizophrenia in terms of adult neurogenesis [11]. Because of the complexity of both the brain and neurobiological markers, more sophisticated systematic approaches are needed to better understand this theory [12]. However, the most widely held theory is the neurobiological one, because of its importance in helping to: a) explain causative mechanisms of schizophrenia, b) identify targets for treatment, and c) predict outcomes [10].

\section{Neurobiological Theory: Understanding Causative Mechanisms and Treatment of Schizophrenia}

\subsection{Onset of Schizophrenia}

Studies that use the neurobiological theory have identified alterations in neurotransmitters which occur at the onset of schizophrenia, helping to explain and predict some inconsistencies in the data. Alterations in neurotransmitter such as dysregulation of dopamine, glutamate, and serotonin, help to explain the onset of schizophrenia [13] [14]. According to the dopamine hypothesis, psychotic symptoms can be developed by drugs that stimulate dopamine activity in non-schizophrenic persons if given in sufficiently high dose whereas psychotic symptoms can be decreased when drugs that block dopamine receptors are given [15]. In addition, since the serotonin-dopamine antagonists have earned a record as having a therapeutic effect, alterations of serotonin and dopamine can be explained as the indicators of onset of schizophrenia [15].

A review of the studies concluded that the identification of abnormalities in the prefrontal cortex in subjects at high risk for schizophrenia was well supported through functional brain imaging studies. The study also found evidence to suggest a number of brain structural abnormalities could be identified as potential markers of schizophrenia. However, there were not strong evidences to support structural abnormalities as illness-related markers in pre-schizophrenic individuals at high risk [16]. Thus, it would seem that findings may change over the course of the illness.

\subsection{Course of Schizophrenia}

Neurobiological theory also helps to predict the course that schizophrenia might take. Every person with schizophrenia may experience different disease progress. However, there are common disease progress patterns. At first, typical psychotic symptoms such as hallucinations or delusions develop as neurotransmitters may not be regulated appropriately. After this acute stage is managed or treated by antipsychotics well, persons with schizophrenia will go through the next stage called as a chronic stage, characte- 
rized by lesser typical psychotic symptoms. However, during this chronic stage, a relapse may occur. Performing structural or functional brain imaging is a method to assess or evaluate the conditions of one's brain. Therefore, through a series of functional brain imaging, prefrontal structural and neurochemical alterations can explain disease progress [17] [18]. In addition, observation of the effects of antipsychotic medications can predict the course of schizophrenia.

\subsection{Relevant Treatment Methods}

Treatment with antipsychotics can be relevant treatment methods according to neurobiological perspective. This drug treatment is currently the most popular way in managing schizophrenia in both clinical and community settings. According to neurobiological theory, schizophrenia is the result of neurotransmitter dysregulation such as: 1) an excess of dopamine, 2) reduced glutamate in the cerebrospinal fluid, 3) levels of gamma aminobutyric acid (GABA) expression in prefrontal cortex, or 4) reduced levels of serotonin [13] [14]. Antipsychotics play a key role in helping to regulate these neurotransmitter abnormalities. Antipsychotics are effective in reducing positive symptoms, such as hallucinations and delusions, and negative symptoms, such as social withdrawal or showing limited emotion. Antipsychotics are usually classified as being one of the two types, namely typical antipsychotics and atypical antipsychotics. For example, haloperidol and phenothiazines, which are typical antipsychotics, block dopamine D2 receptors, resulting in a decrease in positive symptoms. Clozapine, olanzapine, risperidone, quetiapine and ziprazidone, which are atypical antipsychotics, are related to serotonin, dopamine, and muscarinic receptors, resulting in decreases in both positive and negative symptoms. These days, atypical antipsychotics are commonly used due to its effectiveness and lower risk of side effects compared to typical antipsychotics [14].

\subsection{Approaches to Care}

Approaches to care that are guided by neurological theory include performing diagnostic procedures, predicting outcomes, identifying objective targets for treatments, and implementing antipsychotics medications. These approaches are performed according to the needs of individuals and their family members. Pre- and post-care for diagnostic procedures should be conducted, and evaluation and prediction of the outcomes should also be conducted. After closely evaluating the condition of a patient, selecting the most appropriate antipsychotic is very important. Therefore, likely course of a patient's recovery should be explained to the patient, as well as the likely side-effects of the prescribed antipsychotics. Psychoeducation regarding etiology and medication management for patients and their family members should also be performed.

\section{Critique of the Neurobiological Theory}

\subsection{Scientific and Evidence-Based Foundation}

Through recent studies using the neurobiological theory to identify the etiology of 
schizophrenia, there have been many key findings which have advanced the scientific basis of the disease. A number of studies have focused on structural brain neuroimaging, identifying the alterations in the prefrontal lobe involved in schizophrenia. The fact that functional connectivity between prefrontal regions may be impaired has been suggested, and reductions in neuronal and membrane integrity have also been shown. Dysregulation of neurotransmitters have also been identified [10]. A study discussed the relationship between symptom severity and cortical and grey matter volumes in schizophrenia. The study suggested that variation in cortical and grey matter volumes indicates separate neurobiological mechanisms underlying the two major symptom domains, positive and negative symptoms, in schizophrenia [19]. The role of the right hemisphere in a person having diminished expressivity was underscored [20]. Neurobiological studies can explain the causal relationships in discovering the etiology of the schizophrenia, whereas neurodevelopmental epidemiological studies cannot show this [21]. Although there are some inconsistencies between the results of studies, interfering with having a precise understanding of pathogenesis, the scientific foundations of schizophrenia have been well-established.

\subsection{Ability to Guide Risk Factor Identification and Prevention}

Neurobiological theory seldom leads to the identification of risk factor. However, neurobiological studies have indicated that pre-psychotic individuals experience alterations in brain structure and function, and then such individuals may move on psychosis [22]. Whereas, neurodevelopmental theory can explain and predict the risk factors influencing the development of the schizophrenia (such as fetal malnutrition, extreme prematurity, or drug exposures) and that these risk factors are preventable, neurobiological theory has limited explanatory power in this regard. Because it focuses on the explanation of the structural, functional, or neurochemical alterations in the brain, there is little known about how to identify risk factors. Moreover, there is little known about the prevention of the disease. The mechanisms influencing the development of the disease are well-described because schizophrenia is defined as a result of abnormal dysfunctions or structure of the brain. However, this theory does not focus on discovering risk factor identification or on disease prevention.

\subsection{Utility in Guiding Treatment}

Neurobiological theory has a high utility in guiding treatment. This theory employs antipsychotics treatment, the most widely used method to manage schizophrenia. Psychopharmacology based on the understanding of the neurophysiology and neurochemistry, especially focusing on neurotransmitters, can guide effective treatment methods for patients who have schizophrenia [23]. Although genetic theory employs treatment methods such as gene therapy or consultation approaches, these methods may have pitfalls because of their being highly technical and expensive. However, drug treatment, guided by the neurobiology theory, is considered to be an effective way of preventing a relapse and relatively inexpensive. 


\subsection{Attention to the Customer's Perspective and Needs}

Neurobiological theory pays little attention to the customer/patient's perspective and needs because this theory views the etiology of the schizophrenia as structural, functional, and neurochemical alterations in the brain. This theory only focuses on the brain, itself, and is based on the biological mechanisms and findings. Moreover, the health care provider's perspective is more prevalent in this theory because it is guided antipsychotics treatments and assesses and evaluates outcomes in patients. However, this theory describes well the etiology of schizophrenia related to patient's symptoms and treatment needs.

\subsection{Cultural and Gender Sensitivity and Appropriateness}

This theory has facility across cultures. Whereas, genetic theory is not entirely generalizable (because the role of several genes has not been established in all ethnic groups), several studies identified that structural and functional alterations in the brain are identified, regardless of one's culture or ethnic group, or gender. However, as current studies focus on the issues of gender difference, there have been some inconsistent findings in the brain features in male versus females with schizophrenia [8]. For example, bilateral hippocampal volume reduction was found to be in males with schizophrenia, but there was no significant reduction in hippocampal volumes in females with schizophrenia compared to females without schizophrenia [24]. These gender issues are still no certain answer.

\subsection{Applicability across the Age Span}

This theory is applicable to all people, regardless of their age. The age of onset of schizophrenia is adolescent or early-adult periods [2]. Schizophrenia is a chronic disease, especially affecting through the elderly. Regardless of one's age, the neurobiological theory employed by researchers has provided consistent etiology. According to the recent studies, they used the subjects between the teens and fifties years old of age, and there were no inconsistent findings in neurobiological markers [16] [19]. Moreover, it is evident when considering that the same kind of drugs, guided by neurochemical theory, was selected for their treatment methods regardless of their age. However, in the case of the neurodevelopmental theory, the relationship of the cause and the effect according to the age is unclear.

\subsection{Ethical Implications}

This theory has little shortcomings in terms of ethical considerations. Genetic theory focuses on hereditability of the schizophrenia and certain gene identification. Treatment methods are gene replacement therapy or genetic consultation. Moreover, neurodevelopmental theory focuses on adverse environmentally exposures during perinatal periods. Thus, a patient's family members may have sort of guilt feelings about the fact that certain genes are predominant to cause schizophrenia and a patient's disease might be preventable only if avoiding perinatal or environmental risks. However, this neuro- 
biological theory may not have ethical conflicts because this explanation is rooted on the abnormalities of the brain, itself.

\subsection{Overall Usefulness as a Framework of Research}

Overall, the neurobiological theory is useful as a framework for research because it has strong scientific evidence and applies across age and cultures. Moreover, the ability to guide treatment methods is predominant. Therefore, it has a strong power in studying antipsychotic medication intervention research studies. For example, diverse racial/ ethnic groups and diverse age groups can be included to examine the effectiveness of medication. Most nursing-related research studies focus on psychosocial aspects, not only neurobiological aspects of the etiology of schizophrenia to study the phenomenon of the disease. Therefore, this theory has limitations because it focuses on the brain, itself, and is based on the biological mechanisms and findings.

\section{Conclusion}

Of the three theories (genetic, neurodevelopmental, and neurobiological theories), which theory is the right answer regarding the etiology of schizophrenia may be difficult, because each theory may emphasize a different aspect of the disease. However, of these, the neurobiological theory is the predominant theory in explaining the etiology of schizophrenia. The neurobiological theory defines schizophrenia as a result of abnormal dysfunctions, or abnormal structures of the brain. This theory: 1) has a strong scientific foundation, 2) has high utility in guiding treatment, and 3) is appropriate regardless of a patient's age or culture. Moreover, there are few ethical considerations regarding its use when compared with other theories; therefore, it's more useful than all others.

\section{References}

[1] American Psychiatric Association (2000) Diagnostic and Statistical Manual of Mental Disorders. 4th Edition, Text Revision (DSM-IV-TR), American Psychiatric Association, Washington DC.

[2] Gourion, D., Goldberger, C., Leroy, S., Bourdel, M.C., Olie, J.P. and Krebs, M.O. (2005) Age at Onset of Schizophrenia: Interaction between Brain-Derived Neurotrophic Factor and Dopamine D3 Receptor Gene Variants. Neuroreport, 16, 1407-1410. http://dx.doi.org/10.1097/01.wnr.0000175245.58708.6b

[3] Preti, A. and Miotto, P. (2005) Genetics, Perinatal Insult and Schizophrenia: The Mechanism Underlying an Increased Prevalence of Perinatal Complications among Individuals with a Diagnosis of Schizophrenia? Current Psychiatry Reviews, 1, 139-150. http://dx.doi.org/10.2174/1573400054065596

[4] Zuchner, S., Roberts, S.T., Speer, M.C. and Beckham, J.C. (2007) Update on Psychiatric Genetics. Genetics in Medicine, 9, 332-340. http://dx.doi.org/10.1097/GIM.0b013e318065a9fa

[5] Sullivan, P.F. (2008) Schizophrenia Genetics: The Search for a Hard Lead. Current Opinion in Psychiatry, 21, 157-160. http://dx.doi.org/10.1097/YCO.0b013e3282f4efde

[6] Rietkerk, T., Boks, M.P., Sommer, I.E., Liddle, P.F., Ophoff, R.A. and Kahn, R.S. (2008) The 
Genetics of Symptom Dimensions of Schizophrenia: Review and Meta-Analysis. Schizophrenia Research, 102, 197-205. http://dx.doi.org/10.1016/j.schres.2008.01.023

[7] Hall, J., Romaniuk, L., McIntosh, A.M., Steele, J.D., Johnstone, E.C. and Lawrie, S.M. (2009) Associative Learning and the Genetics of Schizophrenia. Trends in Neurosciences, 32, 359-365. http://dx.doi.org/10.1016/j.tins.2009.01.011

[8] Taylor, R. and Langdon, R. (2006) Understanding Gender Differences in Schizophrenia: A Review of the Literature. Current Psychiatry Reviews, 2, 255-265. http://dx.doi.org/10.2174/157340006776875987

[9] Schlotz, W. and Phillips, D.I. (2009) Fetal Origins of Mental Health: Evidence and Mechanisms. Brain Behavior and Immunity, 23, 905-916. http://dx.doi.org/10.1016/j.bbi.2009.02.001

[10] Keshavan, M.S., Tandon, R., Boutros, N.N. and Nasrallah, H.A. (2008) Schizophrenia, "Just the Facts": What We Know in 2008 Part 3: Neurobiology. Schizophrenia Research, 106, 89-107. http://dx.doi.org/10.1016/j.schres.2008.07.020

[11] Reif, A., Schmitt, A., Fritzen, S. and Lesch, K.P. (2007) Neurogenesis and Schizophrenia: Dividing Neurons in a Divided Mind? European Archives of Psychiatry and Clinical Neurosciences, 257, 290-299. http://dx.doi.org/10.1007/s00406-007-0733-3

[12] Bender, W., Albus, M., Moller, H.J. and Tretter, F. (2006) Towards Systemic Theories in Biological Psychiatry. Pharmacopsychiatry, 39, S4-S9.

http://dx.doi.org/10.1055/s-2006-931482

[13] Goff, D.C. (2005) Pharmacologic Implications of Neurobiological Models of Schizophrenia. Harvard Review of Psychiatry, 13, 352-359. http://dx.doi.org/10.1080/10673220500433262

[14] Wong, A.H. and Van Tol, H.H. (2003) Schizophrenia: From Phenomenology to Neurobiology. Neuroscience \& Biobehavioral Reviews, 27, 269-306. http://dx.doi.org/10.1016/S0149-7634(03)00035-6

[15] Sadock, B. and Sadock, V. (2007) Synopsis of Psychiatry. Lippincott, Williams \& Wilkins, Philadelphia.

[16] Pantelis, C., Yucel, M., Bora, E., Fornito, A., Testa, R., Brewer, W.J., Velakoulis, D. and Wood, S.J. (2009) Neurobiological Markers of Illness Onset in Psychosis and Schizophrenia: The Search for a Moving Target. Neuropsychology Review, 19, 385-398.

http://dx.doi.org/10.1007/s11065-009-9114-1

[17] Prasad, K.M., Sahni, S.D., Rohm, B.R. and Keshavan, M.S. (2005) Dorsolateral Prefrontal Cortex Morphology and Short-Term Outcome in First-Episode Schizophrenia. Psychiatry Research, 140, 147-155. http://dx.doi.org/10.1016/j.pscychresns.2004.05.009

[18] Wood, S.J., Berger, G.E., Lambert, M., Conus, P., Velakoulis, D., Stuart, G.W., Desmond, P., McGorry, P.D. and Pantelis, C. (2006) Prediction of Functional Outcome 18 Months after a First Psychotic Episode: A Proton Magnetic Resonance Spectroscopy Study. Archives of General Psychiatry, 63, 969-976. http://dx.doi.org/10.1001/archpsyc.63.9.969

[19] Nesvag, R., Saetre, P., Lawyer, G., Jonsson, E.G. and Agartz, I. (2009) The Relationship between Symptom Severity and Regional Cortical and Grey Matter Volumes in Schizophrenia. Progress in Neuropsychopharmacology and Biological Psychiatry, 33, 482-490. http://dx.doi.org/10.1016/j.pnpbp.2009.01.013

[20] Cohen, A.S., Iglesias, B. and Minor, K.S. (2009) The Neurocognitive Underpinnings of Diminished Expressivity in Schizotypy: What the Voice Reveals. Schizophrenia Research, 109, 38-45. http://dx.doi.org/10.1016/j.schres.2009.01.010

[21] McGrath, J.J. and Richards, L.J. (2009) Why Schizophrenia Epidemiology Needs Neurobiology and Vice Versa. Schizophrenia Bulletin, 35, 577-581. 
http://dx.doi.org/10.1093/schbul/sbp004

[22] Nelson, B., Fornito, A., Harrison, B.J., Yucel, M., Sass, L.A., Yung, A.R., Thompson, A., Wood, S.J., Pantelis, C. and McGorry, P.D. (2009) A Disturbed Sense of Self in the Psychosis Prodrome: Linking Phenomenology and Neurobiology. Neuroscience \& Biobehavioral Review, 33, 807-817. http://dx.doi.org/10.1016/j.neubiorev.2009.01.002

[23] Tretter, F. and Scherer, J. (2006) Schizophrenia, Neurobiology and the Methodology of Systemic Modeling. Pharmacopsychiatry, 39, S26-S35.

http://dx.doi.org/10.1055/s-2006-931486

[24] Exner, C., Nehrkorn, B., Martin, V., Huber, M., Shiratori, K. and Rief, W. (2008) Sex-Dependent Hippocampal Volume Reductions in Schizophrenia Relate to Episodic Memory Deficits. Journal of Neuropsychiatry\& Clinical Neurosciences, 20, 227-230.

http://dx.doi.org/10.1176/jnp.2008.20.2.227

\section{Abbreviation Note}

GWAS: Genome-wide association studies.

COMT: Catechol-O-methyl transferase.

DISC1: Disrupted in schizophrenia 1.

NRG1: Neuregulin 1.

GABA: Gamma aminobutyric acid.

Submit or recommend next manuscript to SCIRP and we will provide best service for you:

Accepting pre-submission inquiries through Email, Facebook, LinkedIn, Twitter, etc. A wide selection of journals (inclusive of 9 subjects, more than 200 journals)

Providing 24-hour high-quality service

User-friendly online submission system

Fair and swift peer-review system

Efficient typesetting and proofreading procedure

Display of the result of downloads and visits, as well as the number of cited articles

Maximum dissemination of your research work

Submit your manuscript at: http://papersubmission.scirp.org/

Or contact ojpsych@scirp.org 